\title{
Wave propagation in a general anisotropic poroelastic medium: Biot's theories and homogenisation theory
}

\author{
M D SHARMA \\ Department of Mathematics, Kurukshetra University, Kurukshetra 136 119, India. \\ e-mail: mohan_here@rediffmail.com
}

\begin{abstract}
Anisotropic wave propagation is studied in a fluid-saturated porous medium, using two different approaches. One is the dynamic approach of Biot's theories. The other approach known as homogenisation theory, is based on the averaging process to derive macroscopic equations from the microscopic equations of motion. The medium considered is a general anisotropic poroelastic (APE) solid with a viscous fluid saturating its pores of anisotropic permeability. The wave propagation phenomenon in a saturated porous medium is explained through two relations. One defines modified Christoffel equations for the propagation of plane harmonic waves in the medium. The other defines a matrix to relate the relative displacement of fluid particles to the displacement of solid particles. The modified Christoffel equations are solved further to get a quartic equation whose roots represent complex velocities of the four attenuating quasi-waves in the medium. These complex velocities define the phase velocities of propagation and quality factors for attenuation of all the quasi-waves propagating along a given phase direction in three-dimensional space. The derivations in the mathematical models from different theories are compared in order to work out the equivalence between them. The variations of phase velocities and attenuation factors with the direction of phase propagation are computed, for a realistic numerical model. Differences between the velocities and attenuations of quasi-waves from the two approaches are exhibited numerically.
\end{abstract}

\section{Introduction}

Wave velocities and attenuation are the two important properties which provide information about the saturation and structure of in situ rocks. It is always important to obtain the relations which relate the propagation properties of a rock to its geophysical properties. The reservoir rocks in the crust can, more closely, be modeled as fluidsaturated porous solids pervaded by aligned cracks. In the presence of aligned cracks, an elastic medium behaves anisotropic to wave propagation (Hudson 1980, 1981). In general, the seismic anisotropy is caused by the lithological and crystal alignments, stress-induced effects, aligned cracks and fluidfilled pores. In the absence of point symmetry in pore distribution, the resulting anisotropy is of a general type with arbitrary symmetry. To design the experiments for reservoir characterisation (Carcione and Quiroga-Goode 1996; Rasolofosaon and Zinszner 2002), a more realistic mathematical model for wave propagation in poroelastic medium is always required. Moreover, the existing theoretical models are not able to explain the seismic attenuation observed in the sedimentary regions (Pride et al 2004).

The dynamic theory for wave propagation in fluid-saturated porous media was developed by Biot (1956). Biot used Lagrange's equations to derive a set of coupled differential equations that govern the motions of solid and fluid phases. Biot (1962a) extended the acoustic propagation theory in the wider context of the mechanics of porous media. Biot (1962b) developed the new features of the extended theory in more detail. This theory is obtained through a new and simplified derivation of

Keywords. Anisotropic poroelastic (APE) solid; Biot's theory; homogenisation theory; phase velocity. 
the fundamental equations of poroelastic propagation. This also provides an exact procedure for the evaluation of the dynamic properties of the fluid motion relative to the solid. The Biot's theory of 1956 and its refined version (Biot 1962a, b) predicted the existence of a highly attenuated second compressional wave along with an expected one. This second wave was verified experimentally by Plona (1980). The agreements between experimental observations and Biot's theories have also been reported in Berryman (1980), Lakes et al (1983) and Plona and Johnson (1984). Klimentos and McCann (1988) studied the experimental observation of Biot's slow wave in natural material. After that, slow wave was detected in air-filled sandstone (Nagy et al 1990) and unconsolidated sand (Boyle and Chotiros 1992). Later, Kelder and Smeulders (1997) observed this wave in water-saturated Nivelsteiner sandstone. Gurevich et al (1999) is a notable experimental study which confirms that the Biot's theory of poroelasticity is adequate to describe the behaviour of porous materials at ultrasonic frequencies. On the other hand, some experimental studies (Winkler 1985; Berryman and Wang 2001) faced disagreement with Biot's theories. King et al (2000) and King and Marsden (2002) studied the velocity dispersion in saturated sandstone samples and discussed the deviations of experimental output from Biot's theory. Such disagreements led to the emergence of alternative approaches to study elastodynamics in the porous solids. One such approach is commonly being used to model the transport processes in porous media. This second approach is based on the homogenisation methods which derive the macroscopic equations by starting with the detailed microscale physical description.

Auriault (1980) used the homogenisation method to develop a new model for elastodynamics in saturated porous solids. This study is based on the macroscopic behaviour of periodic elastic structures embedded by the pores filled with a Newtonian fluid. The pore size is assumed small compared to the size of the macroscopic body. Burridge and Keller (1981) used this two-scale homogenisation approach to derive equations to govern the behaviour of fluid-saturated porous solids. For small viscosity of pore fluid, these equations derived from microstructure were reducible to those of Biot. In this approach, the wave propagation is represented by a pair of differential equations (Auriault et al 1985) in terms of solid displacement and fluid pressure, whereas Biot's equations were in terms of displacements of both the solid and fluid phases. Homogenisation has proven that Biot's model is not the only mathematical model for describing the acoustics of elastic porous media saturated by a viscous Newtonian fluid (Auriault 1991). According to Auriault et al (1985), Biot's approach does not lead to numerical values for the effective coefficients involved in the equations describing the macroscopic behaviour. The essential problem (Boutin et al 1987) is with the values to be given to the viscous, elastic and inertial coupling parameters used in the theory. On the other hand, the homogenisation process enables the computation of all the effective coefficients used in its derivations. However, it is noted that the phenomenological (or macroscopic) approach of Biot is preferred over the homogenisation theory for some studies (Kaynia and Banerjee 1993; Chen 1994). Biot's theory may also be more suitable in the studies involving the conditions on the fluid discharge out of the surface of porous solid matrix. The wave propagation studies across/along the porous-porous interface or fluid-porous interface belong to this category.

Recently, author Sharma (2004, 2005) used Biot's theories to study the wave propagation in a general anisotropic porous solid with anisotropic permeability controlling the flow of viscous fluid in its pores. In another paper, author Sharma (2006) studied the wave propagation in anisotropic thermoelastic medium. This study shows that the mathematical model of thermoelastic propagation is equivalent to a simple mathematical model of poroelastic propagation derived through the homogenisation approach. Thus, the homogenisation model may be preferred to establish a correspondence between the characteristics of poroelastic and thermoelastic propagation. This implies that homogenisation model for poroelastic propagation is as important as Biot's model. Equivalence between these models for isotropic medium has been discussed earlier (Boutin et al 1987). But in anisotropic medium, the constitutive equations of these models differ on the anisotropy of inertial coupling between the constituents of porous aggregates.

The work presented is a comparative study that explores an equivalence between the mathematical models of wave propagation derived from Biot's theories as well as homogenisation theory. Each mathematical model consists of two systems, one relating the displacements of fluid and solid particles and the other is modified Christoffel equations for the medium. The corresponding derivations in these systems are compared in order to establish the possible relations among the parameters of these theories. Relation is also obtained between the dissipation parameters of the models. The difference between the Christoffel equations implies the different propagation characteristics. 
A numerical example calculates the phase velocities and attenuations of all the four quasi-waves in a reservoir rock, using the homogenisation theory and the two theories of Biot. The comparative variations of phase velocities and attenuation coefficients with the phase direction are exhibited for the numerical model.

\section{Anisotropic poroelastic propagation}

\subsection{Biot (1962a, b) theory}

Following Biot (1962a, b), a set of differential equations governs the particle motion in a general anisotropic porous solid frame saturated by a viscous fluid in its pores of anisotropic permeability. These equations, in the absence of body forces, are given by

$$
\begin{gathered}
c_{i j k l}^{\prime} u_{k, j l}+m_{i j} w_{k, j k}=\rho \ddot{u}_{i}+\rho_{f} \ddot{w}_{i}, \\
m_{k j} u_{k, i j}+R w_{k, i k}=\rho_{f} \ddot{u}_{i}+q_{i j} \ddot{w}_{j}+\mu r_{i j} \dot{w}_{j},
\end{gathered}
$$

where $c_{i j k l}^{\prime}$ are elastic constants of the porous aggregate. The coefficient $R$ measures the pressure required on the fluid to force a certain volume of it into the porous aggregate while the total volume remains constant. The $u_{i}$ are the components of the average displacements for the solid and $w_{i}$ are the components of displacement of fluid relative to the solid. Indices can take the values 1,2 and 3 . Summation convention is valid for repeated indices. The comma (,) before an index represents partial space differentiation and dot over a variable represents partial time derivative. The $\rho$ and $\rho_{f}$ are the densities of porous aggregate and pore fluid, respectively. The symmetric matrices $\mathbf{M}=\left\{m_{i j}\right\}$ and $\mathbf{q}=\left\{q_{i j}\right\}$ of elastic and inertial parameters, respectively control the anisotropic coupling between fluid and solid phases. Another tensor $r_{i j}$ steers the generalized Darcy's law for fluid-solid coupling and represents the inverse of anisotropic permeability $\left(\chi_{i j}\right)$ tensor. All these tensors are symmetric. Depending upon frequency $(\omega)$, viscosity $(\mu)$ and permeability tensor $\chi\left(=\chi_{o} \chi_{a}\right)$, dissipation is represented by a matrix

$$
\mathbf{d}=\imath \frac{\mu}{\omega \rho_{f} \chi_{o}}\left\{\chi_{\mathbf{a}}\right\}^{-1}
$$

This expression of $\mathbf{d}$ is valid only in the lowfrequency range that ensures Poiseuille flow in pores. For higher frequencies, a correction factor (Biot 1956) is applied to the viscosity $\mu$, replacing it by $\mu F(\kappa)$. The relevant asymptotic expressions are the same as in Sharma (2005).
To seek the harmonic solution of (1), for the propagation of plane waves, write

$$
\begin{array}{ll}
u_{j}=S_{j} \exp \left\{i \omega\left(p_{k} x_{k}-t\right)\right\} ; & (j=1,2,3), \\
w_{j}=F_{j} \exp \left\{i \omega\left(p_{k} x_{k}-t\right)\right\}, & (j=1,2,3),
\end{array}
$$

where $\omega$ is angular frequency and $\left(p_{1}, p_{2}, p_{3}\right)$ is slowness vector. In terms of phase velocity $v$, the slowness $\left(p_{1}, p_{2}, p_{3}\right)=\mathbf{N} / v$ so that the row matrix $\mathbf{N}=\left(n_{1}, n_{2}, n_{3}\right)$ represents the direction of phase propagation. Substituting (3) in (1), yields a system of six homogeneous equations in $S_{1}, S_{2}, S_{3}, F_{1}, F_{2}, F_{3}$. Following Sharma (2005), the modified Christoffel equations, for the propagation of harmonic waves in a general APE medium are given by (for $h=v^{2}$ )

$$
\begin{aligned}
& W_{i j} S_{j}=0, \quad(i=1,2,3) \\
& \mathbf{W}=\mathbf{D}_{1} h+\mathbf{D}_{2}+\frac{\mathbf{D}_{3}}{e_{0} h+e_{1}}+\frac{\mathbf{D}_{4}}{h\left(e_{0} h+e_{1}\right)},
\end{aligned}
$$

where coefficient matrices are

$$
\begin{aligned}
& \mathbf{D}_{\mathbf{1}}=-\rho \mathbf{I}+\frac{1}{e_{0}} \mathbf{X}_{\mathbf{1}} ; \\
& \mathbf{D}_{\mathbf{2}}=\mathbf{Z}^{\prime}+\frac{1}{e_{0}}\left(\mathbf{X}_{\mathbf{2}}-\frac{e_{1}}{e_{0}} \mathbf{X}_{\mathbf{1}}\right) ; \\
& \mathbf{D}_{\mathbf{3}}=\mathbf{X}_{\mathbf{3}}-\frac{e_{1}}{e_{0}}\left(\mathbf{X}_{\mathbf{2}}-\frac{e_{1}}{e_{0}} \mathbf{X}_{\mathbf{1}}\right) ; \\
& \mathbf{D}_{\mathbf{4}}=\frac{R}{\rho_{f}} \mathbf{M N}^{\prime} \mathbf{N} \mathbf{\Psi} \mathbf{N}^{\prime} \mathbf{N M} .
\end{aligned}
$$

The matrix $\mathbf{Z}^{\prime}=\left\{c_{i j k l}^{\prime} n_{j} n_{l}\right\}$ and $e_{0}=\rho_{f} d_{0}$. The symmetric matrix $\boldsymbol{\Psi}$ and other variables (i.e., $d_{0}, e_{1}$, and $\mathbf{X}_{\mathbf{j}}$ ) are the same as expressed in Sharma (2005).

The two forms of displacements, i.e., (w) and $(\mathbf{u})$, are related by

$$
\begin{aligned}
w_{i}= & \Gamma_{i j} u_{j}, \quad(i=1,2,3) ; \\
\boldsymbol{\Gamma}= & \frac{1}{e_{0} h+e_{1}}\left[-\rho_{f} \mathbf{\Phi} h+\mathbf{\Phi} \mathbf{N}^{\prime} \mathbf{N} \mathbf{M}-R \boldsymbol{\Psi}\right. \\
& \left.+\frac{R}{h \rho_{f}} \mathbf{\Psi} \mathbf{N}^{\prime} \mathbf{N M}\right] .
\end{aligned}
$$

\subsection{Biot (1956) theory}

From the previous sub-section, it may be noted that the modified Christoffel equations (4) and 
relation (6) between the displacements of constituents explain the wave propagation in the anisotropic porous material. Using Biot (1956) theory, the matrix $\boldsymbol{\Gamma}$ relating the displacements $\mathbf{u}$ and $\mathbf{w}$ is expressed as

$$
\begin{aligned}
\boldsymbol{\Gamma}= & \frac{f}{e_{0} h+e_{1}}\left[-\left(e_{0} \mathbf{I}+\rho_{12} \boldsymbol{\Phi}-\rho_{22} \mathbf{\Phi} \mathbf{d}\right) h\right. \\
& +\left(\mathbf{\Phi} \mathbf{N}^{\prime} \mathbf{N} \mathbf{M}+R \mathbf{\Psi} \mathbf{d}-R r_{12} \boldsymbol{\Psi}-e_{1} \mathbf{I}\right) \\
& \left.+\frac{R}{h \rho_{22}} \boldsymbol{\Psi} \mathbf{N}^{\prime} \mathbf{N} \mathbf{M}\right]
\end{aligned}
$$

where $e_{0}=\rho_{22} d_{0}, \mathbf{M}=\left\{m_{i j}\right\}, h=v^{2}, r_{12}=\rho_{12} /$ $\rho_{22}$. The $d_{0}, e_{1}$ and matrices $\boldsymbol{\Phi}, \quad \boldsymbol{\Psi}$ are expressed as in the previous sub-section except that the matrix $\mathbf{Y}$ used to define matrix $\Psi$ is $\mathbf{I}+\mathbf{d}$ instead of $\mathbf{Y}=\left(1 / \rho_{f}\right) \mathbf{q}+\mathbf{d}$. The dissipation matrix $\mathbf{d}=\imath\left(\mathbf{b} / \omega \rho_{22}\right)$. The definitions of various parameters/constants used are the same as in Sharma (2004). The Christoffel equations, similar to (4), are also obtained from Sharma (2004).

\subsection{Homogenisation theory}

Following Boutin et al (1987), the governing equations for anisotropic fluid-saturated porous media, in the absence of body forces are

$$
\begin{aligned}
\left(c_{i j k l} u_{k, l}-\alpha_{i j} P\right)_{, j} & =\rho \ddot{u}_{i}+\rho_{f} \Theta_{i j}\left(\rho_{f} \omega^{2} \ddot{u}_{j}+\omega^{2} P_{, j}\right), \\
\alpha_{j k} u_{j, k}+\beta P & =-\Theta_{j k}\left(\rho_{f} \omega^{2} u_{j, k}-P_{, j k}\right),
\end{aligned}
$$

where $c_{i j k l}$ are elastic constants and $\alpha_{i j}\left(=\alpha_{j i}\right)$ are effective stress coefficients. The $\beta$ represents the compressibility of the aggregate. The $\rho$ and $\rho_{f}$ are the densities of porous aggregate and pore fluid, respectively. The tensor $\Theta_{j k}$ steers the generalized Darcy's law for fluid-solid coupling. Depending upon frequency $(\omega)$, viscosity $(\mu)$ and permeability tensor $\left(\chi=\chi_{o} \chi_{a}\right)$, this matrix is expressed as

$$
\boldsymbol{\Theta}=-\left(\frac{m}{f} \omega^{2} \rho_{f} \mathbf{I}+\imath \frac{\mu}{\chi_{o}} \omega\left\{\chi_{a}\right\}^{-1}\right)^{-1}
$$

where $\mathbf{I}$ is identity matrix of third order, $m$ is tortuosity coefficient and $f$ is porosity. The equation (9) is a macroscopic description and will not be valid for large frequency $(\omega)$. For high frequencies, the asymptotic approximations are discussed in Auriault et al (1985). In the equations of motion (8), the $u_{i}$ are the components of the average displacements for the solid and $P$ is the fluid pressure in pores. The definitions of dummy index, dot and comma notations for equations (1)-(2) are valid. The system (8) is rewritten as

$$
\begin{aligned}
& c_{i j k l} u_{k, j l}-\hat{\alpha}_{i j} P_{, j}=\hat{\rho}_{i k} \ddot{u}_{k}, \\
& \hat{\alpha}_{j k} u_{j, k}=-\beta P+\Theta_{j k} P_{, j k},
\end{aligned}
$$

where $\hat{\rho}_{i j}=\rho \delta_{i j}+\rho_{f}^{2} \omega^{2} \Theta_{i j},(i, j=1,2,3)$ and $\hat{\alpha}_{i j}=\alpha_{i j}+\rho_{f} \omega^{2} \Theta_{i j},(i, j=1,2,3)$.

This set of differential equations governs the particle motion in a general anisotropic porous solid frame saturated by a viscous fluid in its pores of anisotropic permeability. The equations (8) to (10) imply that, in homogenisation approach, the anisotropic inertial coupling is hiding in the tensor $\left\{\hat{\rho}_{i j}\right\}$. Another tensor of non-dimensional stress coefficients $\hat{\alpha}_{i j}$ represents the elastic coupling between the two constituents of porous aggregate. These coefficients represent the effect of fluid pressure on the motion of solid particles and vice-versa. Whereas in Biot's theories, elastic parameters $m_{i j}$ are used to represent the elastic coupling through dilatations. Thus, the representations of fluid-solid coupling in homogenisation theory is different from those used in the theories of Biot.

A harmonic solution for plane wave propagation given by

$$
\begin{aligned}
u_{j} & =S_{j} \exp \left\{i \omega\left(p_{k} x_{k}-t\right)\right\} ; \quad(j=1,2,3), \\
P & =P_{o} \exp \left\{i \omega\left(p_{k} x_{k}-t\right)\right\},
\end{aligned}
$$

provides (Sharma 2006) the modified Christoffel equations similar to equations (4) but with

$$
\begin{aligned}
& \mathbf{D}_{1}=-\hat{\rho} ; \quad \mathbf{D}_{2}=\mathbf{Z}+\frac{1}{\beta} \hat{\alpha} \mathbf{N}^{\prime} \mathbf{N} \hat{\alpha} ; \\
& \mathbf{D}_{3}=-\frac{\psi \omega^{2}}{\beta} \hat{\alpha} \mathbf{N}^{\prime} \mathbf{N} \hat{\alpha} ; \quad \mathbf{D}_{4}=0 ; \\
& e_{0}=\beta ; \quad e_{1}=\psi \omega^{2}, \quad\left(\psi=\mathbf{N} \Theta \mathbf{N}^{\prime}\right) .
\end{aligned}
$$

The matrix $\mathbf{Z}=\left\{c_{i j k l} n_{j} n_{l}\right\}$. The expression (Auriault et al 1985)

$$
w_{i}=\Theta_{i j}\left(\rho_{f} \omega^{2} u_{j}-P_{, j}\right)
$$

is used to relate the relative displacement of fluid phase (w) and solid phase (u) by

$$
\begin{aligned}
& w_{i}=\Gamma_{i j} u_{j}, \quad(i=1,2,3) \\
& \boldsymbol{\Gamma}=\omega^{2} \boldsymbol{\Theta}\left[\rho_{f} \mathbf{I}-\frac{\mathbf{N}^{\prime} \mathbf{N} \hat{\alpha}}{\beta h+\psi \omega^{2}}\right] .
\end{aligned}
$$

\section{Phase velocity and attenuation}

For the non-trivial solution of the modified Christoffel equations, the determinant of matrix $W$ 
must vanish. This is solved into a biquadratic equation in $h\left(=v^{2}\right)$, given by

$$
h^{4}-c_{1} h^{3}+c_{2} h^{2}-c_{3} h+c_{4}=0 .
$$

The coefficients in this polynomial equation are derived (Sharma 2005) from $e_{0}, e_{1}$ and $D_{j}$, $(j=1,2,3,4)$. These coefficients are complex even for real $\mathbf{N}$. This implies that four roots of this equation may be complex. Let $h_{j},(j=1,2,3,4)$, denote the four roots of this equation. The complex phase velocities of the quasi-waves, given by $v_{j}\left(=\sqrt{h_{j}}\right)$ will be varying with the direction of phase propagation. The complex velocity of a quasi-wave ' $j$ ', i.e., $v_{j}=v_{R}+\imath v_{I}$, defines the phase propagation velocity $V_{j}=\left(v_{R}^{2}+v_{I}^{2}\right) / v_{R}$ and attenuation quality factor $Q_{j}^{-1}=-2 v_{I} / v_{R}$ for the corresponding wave. Therefore, the four waves propagating in such a medium are attenuating waves. The real $\mathbf{N}$ defines the same direction for propagation vector and attenuation vector and hence, these waves are homogeneous waves. Unlike the waves in isotropic media, polarizations of these waves in anisotropic media may not be along the dynamical axes (i.e., axes of the orthogonal coordinate system with one of them along the direction of propagation). Hence, these waves are called quasi-waves (Crampin 1989). Analogous to the propagation in an isotropic poroelastic medium, these waves represented by $j=1,2,3,4$ may be called the $q P 1-, q P 2-, q S 1-, q S 2$-waves, respectively.

\section{Comparison of the theories}

From the previous sections, it may be noted that the wave propagation phenomenon can be explained through two systems of equations. One is a system of three homogeneous (modified Christoffel) equations, given by (for $h=v^{2}$ )

$$
\left[\mathbf{D}_{\mathbf{1}} h+\mathbf{D}_{\mathbf{2}}+\frac{\mathbf{D}_{3}}{e_{0} h+e_{1}}+\frac{\mathbf{D}_{\mathbf{4}}}{h\left(e_{0} h+e_{1}\right)}\right] \mathbf{S}=0 .
$$

The other is a relation between the solid displacement $(\mathbf{u})$ and relative fluid displacement $(\mathbf{w})$, given by

$$
w_{i}=\Gamma_{i j} u_{j}, \quad(i=1,2,3) .
$$

The expressions for the matrix $\boldsymbol{\Gamma}$ and coefficients D's are different for all the theories. The compatibility among these expressions may define the conditions for the equivalence of these theories. To start with, a reduced and simple case may be more suitable.

\subsection{Non-dissipative case}

The interstitial fluid is assumed as a non-viscous one, i.e., viscosity $\mu=0$. The whole system becomes independent of frequency. For easy comparison, the inertial matrix $\mathbf{q}$ in Biot (1962a, b) theory is assumed isotropic, i.e., $\mathbf{q}=q \mathbf{I}$. The matrix $\Gamma$ in different theories is expressed as follows.

Biot (1956) theory:

$$
\begin{aligned}
\boldsymbol{\Gamma}= & \frac{f}{\rho_{22} h-R}\left[-\left(\rho_{12}+\rho_{22}\right) h \mathbf{I}\right. \\
& \left.+\mathbf{N}^{\prime} \mathbf{N} \mathbf{M}+\left(1+r_{12}\right) R \mathbf{I}-R r_{12} \mathbf{N}^{\prime} \mathbf{N}\right] .
\end{aligned}
$$

Biot (1962a, b) theory:

$$
\begin{aligned}
\boldsymbol{\Gamma}= & \frac{1}{q h-R} \\
& \times\left[-\rho_{f} h \mathbf{I}+\mathbf{N}^{\prime} \mathbf{N} \mathbf{M}+\frac{R}{q} \rho_{f}\left(\mathbf{I}-\mathbf{N}^{\prime} \mathbf{N}\right)\right] .
\end{aligned}
$$

Homogenisation theory:

$$
\begin{aligned}
\Gamma= & \frac{f}{\left(m \rho_{f} \beta h-f\right)} \\
& \times\left[-\rho_{f} \beta h \mathbf{I}+\mathbf{N}^{\prime} \mathbf{N} \alpha+\frac{f}{m}\left(\mathbf{I}-\mathbf{N}^{\prime} \mathbf{N}\right)\right] .
\end{aligned}
$$

The expressions of $\boldsymbol{\Gamma}$ from the two theories of Biot are compared to obtain

$$
\begin{aligned}
\rho_{22} & =q f^{2}, \rho_{12}=f \rho_{f}-q f^{2}, \\
\rho_{11} & =\rho-2 f \rho_{f}+q f^{2}, \quad R^{(1)}=f^{2} R^{(2)}, \\
\mathbf{M}^{(1)} & =f\left(\mathbf{M}^{(2)}-f R^{(2)} \mathbf{I}\right),
\end{aligned}
$$

where the superscripts (1) and (2) correspond to Biot theories of 1956 and 1962, respectively. These relations are similar to those derived in Biot (1962a). Similarly, the comparison of expressions from Biot (1956) theory and homogenisation theory results in

$$
\begin{aligned}
\rho_{22} & =m f \rho_{f}, \quad \rho_{12}=(1-m) f \rho_{f}, \\
\rho_{11} & =\rho-(2-m) f \rho_{f}, \quad R^{(1)}=\frac{f^{2}}{\beta}, \\
\mathbf{M}^{(1)} & =\frac{f}{\beta}(\alpha-f \mathbf{I}) .
\end{aligned}
$$

These relations for isotropic medium have also been obtained earlier (Boutin et al 1987). The $\boldsymbol{\Gamma}$ of homogenisation theory can also be obtained from 
that in Biot (1962a, b) theory. This is achieved with the substitutions of

$$
q=\frac{m}{f} \rho_{f} ; \quad R=\frac{1}{\beta} ; \quad \mathbf{M}=\frac{\alpha}{\beta} .
$$

The above equalities ensure that the relation between the displacements of two constituents of porous aggregate is the same in all the theories.

The propagation phenomenon in the medium is, in fact, governed by the Christoffel equations $(\mathbf{W} S=0)$. In this part of comparison, the coefficient matrices $\mathbf{W}$ are obtained for the nondissipative porous medium. These are explained as follows.

Biot (1956) theory:

$$
\begin{aligned}
\mathbf{W}= & \rho_{22}\left(r_{12}^{2}-r_{11}\right) \mathbf{I} h+(\mathbf{Z}+\mathbf{X}) \\
& +\frac{1}{\rho_{22} h-R}\left(\mathbf{M} \mathbf{N}^{\prime} \mathbf{N M}+R \mathbf{X}\right) \\
\mathbf{X}= & r_{12}\left[R r_{12} \mathbf{N}^{\prime} \mathbf{N}-\left(\mathbf{M N}^{\prime} \mathbf{N}+\mathbf{N}^{\prime} \mathbf{N M}\right)\right]
\end{aligned}
$$

Biot (1962a, b) theory:

$$
\begin{aligned}
\mathbf{W}= & \left(-\rho+\frac{1}{q} \rho_{f}^{2}\right) \mathbf{I} h \\
& +\left[\mathbf{Z}+f\left(\mathbf{M} \mathbf{N}^{\prime} \mathbf{N}+\mathbf{N}^{\prime} \mathbf{N M}-f R \mathbf{N}^{\prime} \mathbf{N}\right)+\mathbf{X}\right] \\
& +\frac{1}{q h-R}\left(\mathbf{M} \mathbf{N}^{\prime} \mathbf{N} \mathbf{M}+R \mathbf{X}\right),
\end{aligned}
$$$$
\mathbf{X}=\frac{\rho_{f}}{q}\left[R \frac{\rho_{f}}{q} \mathbf{N}^{\prime} \mathbf{N}-\left(\mathbf{M N}^{\prime} \mathbf{N}+\mathbf{N}^{\prime} \mathbf{N M}\right)\right] .
$$

Homogenisation theory:

$$
\begin{aligned}
\mathbf{W}= & \left(-\rho+\frac{f}{m} \rho_{f}\right) h \mathbf{I}+\left(\mathbf{Z}+\mathbf{X}+\frac{1}{\beta} \alpha \mathbf{N}^{\prime} \mathbf{N} \alpha\right) \\
& +\frac{f}{\beta\left(m \rho_{f} \beta h-f\right)}\left(\alpha \mathbf{N}^{\prime} \mathbf{N} \alpha+\mathbf{X}\right), \\
\mathbf{X}= & \frac{f}{m}\left[\frac{f}{m} \mathbf{N}^{\prime} \mathbf{N}-\left(\alpha \mathbf{N}^{\prime} \mathbf{N}+\mathbf{N}^{\prime} \mathbf{N} \alpha\right)\right] .
\end{aligned}
$$

It is worked out that the equalities (18) to (20) are not sufficient enough to make these expressions the same. However, using (18), the matrix $\mathbf{W}$ for Biot (1962a, b) theory can be obtained from that for Biot (1956) theory but only with isotropic inertial matrix $\mathbf{q}=q \mathbf{I}$. Otherwise, each of the theories may yield different characteristics of wave propagation in saturated poroelastic medium.

It may be noted that the presence of the term $\alpha \mathbf{N}^{\prime} \mathbf{N} \alpha$ in the coefficient $\mathbf{D}_{\mathbf{2}}$ of homogenisation theory makes its $\mathbf{W}$ dissimilar from those in Biot's theories. So the three expressions of $\mathbf{W}$ cannot be made the same unless some restrictions are applied on matrix $\mathbf{M}=\left\{m_{i j}\right\}$ of Biot's theories and matrix $\alpha$ of homogenisation theory. This implies that the difference in these theories comes as expected, from the elastic coupling between fluid and solid phases. The implied result is that the Christoffel equations from homogenisation theory are not similar to those from the Biot's theories. Thus, despite keeping similar relation between displacement of solid and fluid phases, these theories yield different solutions for phase velocities, attenuations and displacements of particles.

\subsection{Dissipative case}

The comparison between the expressions of $\Gamma$ in different theories is no longer important because the characteristics of propagation are governed by the Christoffel equations. The discussion in the previous section may be generalized to conclude that Christoffel equations of these theories may not be compatible in the presence of dissipation. The coefficient $\mathbf{D}_{4}=0$ in the homogenisation model implies that Biot's theories provide more general Christoffel equations for wave propagation in poroelastic solids. The presence of dissipation is represented by the dissipation matrices. So, the relations among these matrices from different theories hold the interest in this case. This is explained as follows.

In Biot (1956) theory, a dissipation matrix $\mathbf{d}$ is defined as

$$
\mathbf{d}^{(1)}=\imath \Delta_{o}\left\{\chi_{\mathbf{a}}\right\}^{-1} ; \quad \Delta_{o}=\left(1+r_{12}\right) \frac{\omega_{c}}{\omega},
$$

where $\omega_{c}$ is called characteristic frequency. The values of frequency $\omega \ll \omega_{c}$ ensures the Poiseuille flow in pores and represent the low-frequency regime of Biot's theory. The other values of frequency $\omega \gg \omega_{c}$ represent the high frequency regime of Biot's theory.

Using the above expression for $\Delta_{o}$, the dissipation matrix of Biot (1962a, b) theory is given by

$$
\mathbf{d}^{(2)}=\imath \frac{\mathbf{q}}{\rho_{f}} \Delta_{o}\left\{\chi_{\mathbf{a}}\right\}^{-1},
$$

so that $\mathbf{d}^{(2)}=\mathbf{q d}^{(1)} / \rho_{f}$ relates the dissipation matrices from two theories of Biot. This relation along with (18) ensures that the two theories of Biot are the same except for the anisotropic inertial coupling in Biot (1962a, b) theory. In the homogenisation method, the dissipation matrix $\boldsymbol{\Theta}$ from Darcy's law is expressed as

$$
\omega^{2} \Theta=-\frac{f}{m \rho_{f}}\left(\mathbf{I}+\imath \Delta_{o}\left\{\chi_{a}\right\}^{-1}\right)^{-1}
$$



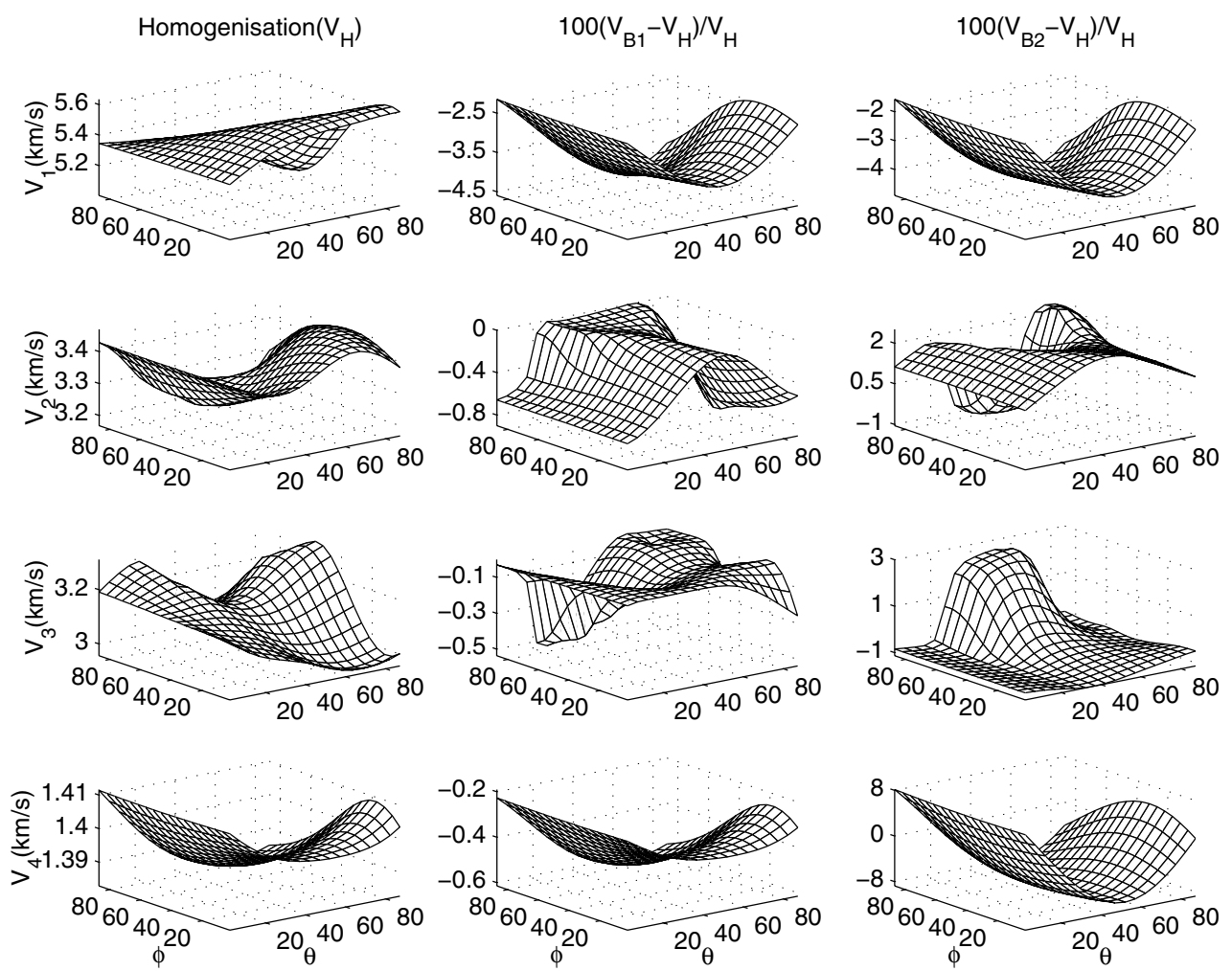

Figure 1. Variations of phase propagation velocities $\left(V_{j} ; j=1,2,3,4\right)$ with the phase direction $(\theta, \phi)$, in non-dissipative anisotropic poroelastic medium (dolomite). H: homogenisation theory; B1: Biot (1956) theory; B2: Biot (1962a, b) theory and angles are in degrees.

and expression $\omega^{2} \Theta=-\left(f / m \rho_{f}\right)\left(\mathbf{I}+\mathbf{d}^{(1)}\right)^{-1}$ relates it to the dissipation in Biot (1956) theory.

In the dissipative porous media, the difference between the Christoffel equations from the different theories can be explained numerically by computing their solutions to define phase velocities and attenuations. The variations of phase velocities and attenuations with the direction of propagation can be compared for each of the four quasi-waves propagating in the medium.

\section{Numerical computation and discussion}

The analytical expressions derived in the previous sections represent a general mathematical model for the wave propagation in a saturated poroelastic solid. These expressions can be used to compute the effects of elastic/hydraulic anisotropy of porous aggregate, viscosity of interstitial fluid, and porosity of solid, on the phase propagation (velocities, attenuations and particle motions). In the present study, the main purpose is to compare Biot's theories and the homogenisation process for wave propagation in poroelastic solids. Hence, the numerical part is restricted to compute the variations of velocities and attenuations of all the four quasi-waves with the direction of phase propagation. For a general direction, given by $(\theta, \phi)$, in three-dimensional space, the row matrix $\mathbf{N}=(\sin \theta \cos \phi, \quad \sin \theta \sin \phi, \quad \cos \theta)$.

Analysis of phase velocities, and attenuations in a real crystal may be a useful study. Elastic matrix (GPa) for Dolomite, an anisotropic reservoir rock (Rasolofosaon and Zinszner 2002), is written as:

$$
\begin{aligned}
& c_{11}=65.53 \quad c_{12}=9.77 \quad c_{13}=12.19 \\
& c_{14}=0.18 Z_{1} \quad c_{15}=-0.81 Z_{1} \quad c_{16}=2.94 Z_{2} ; \\
& c_{22}=50.77 \quad c_{23}=11.61 \quad c_{24}=-0.09 Z_{1} \\
& c_{25}=-0.50 Z_{1} \quad c_{26}=-0.19 Z_{2} ; \\
& c_{33}=60.11 \quad c_{34}=-1.61 Z_{1} \quad c_{35}=1.78 Z_{1} \\
& c_{36}=0.84 Z_{2} ; \\
& c_{44}=23.51 \quad c_{45}=1.49 Z_{2} \quad c_{46}=-1.17 Z_{1} ; \\
& c_{55}=24.57 \quad c_{56}=0.26 Z_{1} ; \\
& c_{66}=20.21 .
\end{aligned}
$$



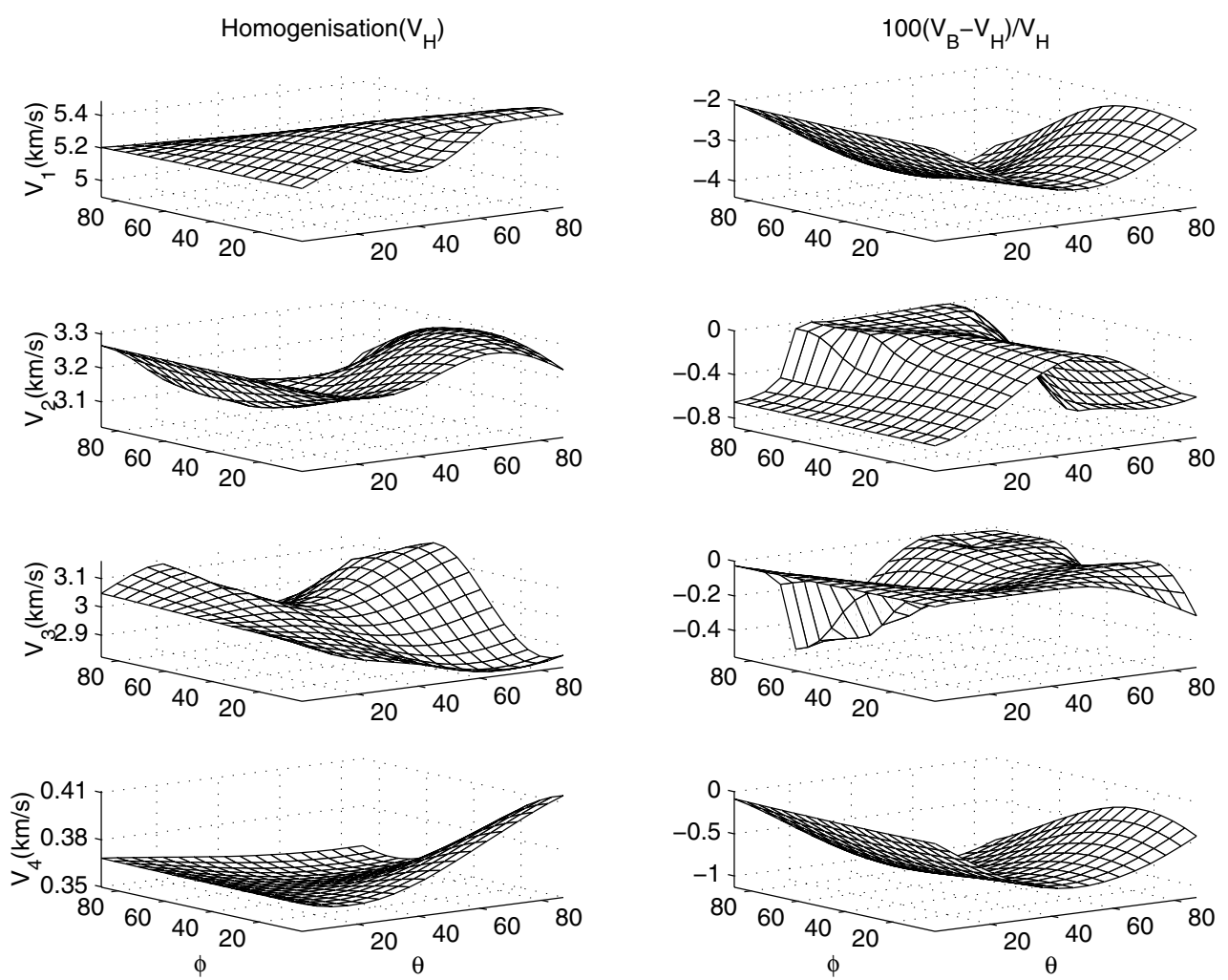

Figure 2. Variations of phase velocities $\left(V_{j} ; j=1,2,3,4\right)$ of quasi-waves with the phase direction $(\theta, \phi)$, in dissipative dolomite. Low frequency: $\Delta_{o}=20$; H: homogenisation theory; B: Biot $(1962 \mathrm{a}, \mathrm{b})$ theory and angles are in degrees.

The symmetric tensor of anisotropic permeability is given by,

$$
\begin{aligned}
\chi_{a}= & \left\{0.96,-0.08 Z_{2},-0.06 Z_{1} ;-0.08 Z_{2}, 0.72,\right. \\
& \left.0.01 Z_{1} ;-0.06 Z_{1}, 0.01 Z_{1}, 0.73\right\} .
\end{aligned}
$$

The values assumed for elastic coupling parameters are $\alpha=0.83 \mathbf{M}_{\mathbf{a}}$ where the anisotropy matrix $\mathbf{M}_{\mathbf{a}}=\left\{1,-0.1 Z_{2}, 0.2 Z_{1} ;-0.1 Z_{2}, 1.1,0.15 Z_{1}\right.$; $\left.0.2 Z_{1}, 0.15 Z_{1}, 0.9\right\}$. The expression $\mathbf{q}=\left(m \rho_{f} / f\right)$ $\mathbf{M}_{\mathbf{a}}$ implies the same anisotropy for inertial coupling matrix q of Biot (1962a, b) theory. The above given set of numerical values defines the triclinic system of anisotropy when $Z_{1}=Z_{2}=1$. The values $Z_{1}=0, Z_{2}=1$ represent the monoclinic symmetry and the values $Z_{1}=Z_{2}=0$ represent the orthorhombic symmetry. A porous aggregate of density $2423 \mathrm{~kg} / \mathrm{m}^{3}$ is containing a $23 \%$ pore space filled with a fluid of density $980 \mathrm{~kg} / \mathrm{m}^{3}$. The assumed values of tortuosity $m=1.05$ and aggregate compressibility $\beta=0.105 / \mathrm{GPa}$, with porosity $f=0.23$ are used in relations $(18)-(20)$ to get the numerical values of parameters $\rho_{11}, \rho_{12}, \rho_{22}, R, \mathbf{M}$ of Biot's theories. The Poiseuille flow $\left(\omega \ll \omega_{c}\right)$ in pores is ensured by the value of $\omega / \omega_{c}<0.1$. This restricts the value of $\Delta_{o}>10\left(1+r_{12}\right)$ for low frequency wave propagation regime of Biot (1956) theory. The expressions of homogenisation theory considered in the present study are not valid for a larger frequency. However, the low frequency regime of Biot's theory contains much of the seismic band of frequencies (say, $1 \mathrm{~Hz}$ to $10 \mathrm{kHz}$ ). Hence, the numerical calculations are restricted only to the low-frequency regime of Biot's theory.

Using the above numerical values, the variations of phase propagation velocities $\left(V_{j}\right)$, attenuation quality factors $\left(100 / Q_{j}\right)$ with the phase direction are computed. The phase direction $(\theta, \phi)$ varies from $(0,0)$ to $\left(90^{\circ}, 90^{\circ}\right)$. The variations are explained as follows.

Figure 1 exhibits the variations of velocities with the phase direction in the anisotropic poroelastic (APE) solid saturated with a non-viscous fluid $\left(\Delta_{o}=0\right)$. These plots may serve as a platform to study the effects of fluid viscosity and solid permeability on the wave propagation. The velocities of $q P 1$-waves from Biot's theories may be 2 to $5 \%$ lower than the velocities obtained by the homogenisation approach. For the other three waves, Biot (1956) theory and homogenisation theory obtain nearly the same velocities. However, it is not the case with Biot $(1962 \mathrm{a}, \mathrm{b})$ theory and velocity of the slowest $(q S 2)$ wave may be different, by 

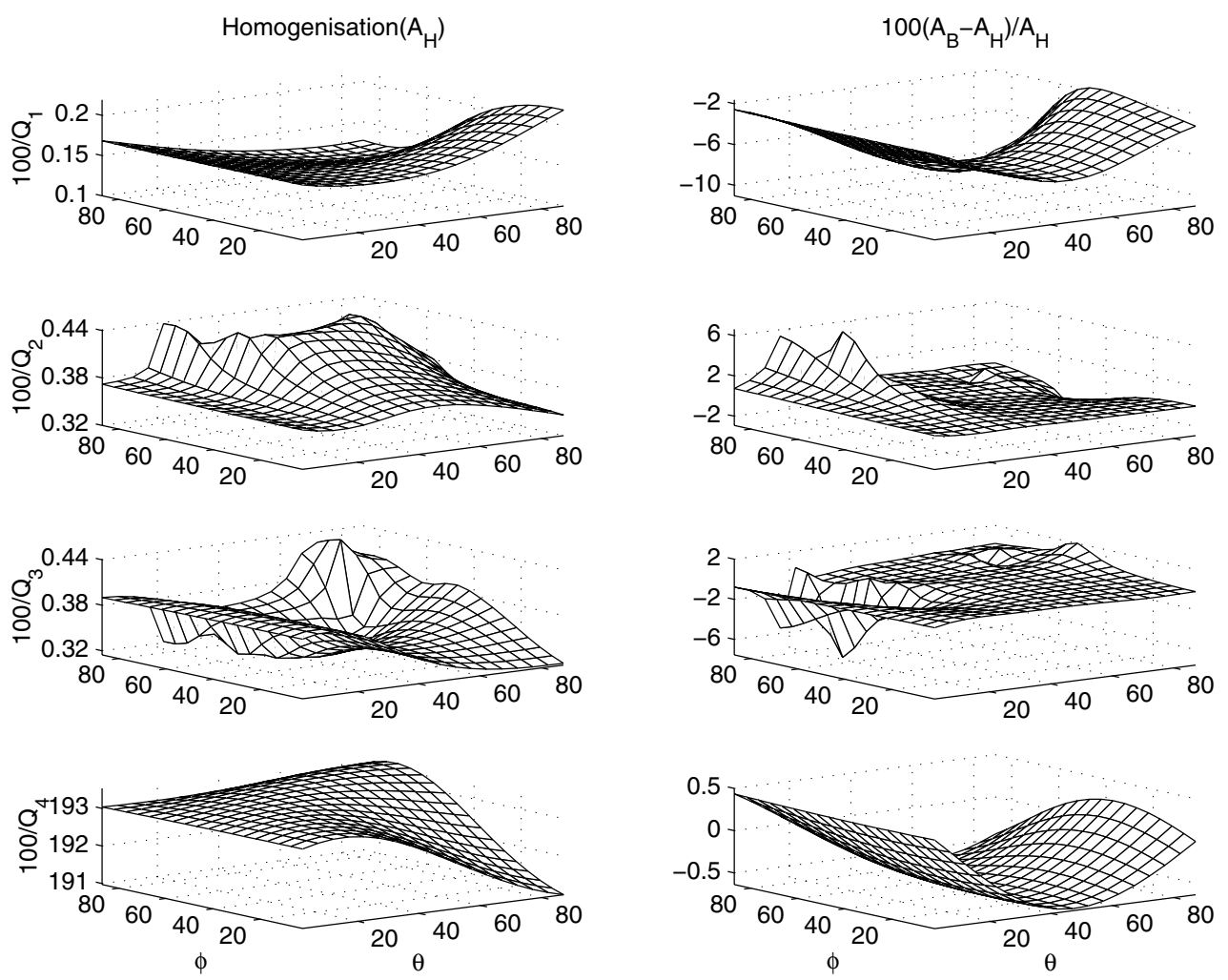

Figure 3. Variations of quality factor $\left(Q_{j} ; j=1,2,3,4\right)$ of quasi-waves with the phase direction $(\theta, \phi)$, in dissipative dolomite. Low frequency: $\Delta_{o}=20 ; A_{k}=100 / Q_{k}, k=\mathrm{H}$ and $\mathrm{B}$ for homogenisation theory and Biot (1962a, b) theory, respectively and angles are in degrees.

about $8 \%$, from that obtained through homogenisation. The velocities of other two $(q P 2, q S 1)$ waves obtained by using Biot (1962a, b) theory are large up to $3 \%$. It may be noted that, in this nondissipative medium, the two theories of Biot obtain the same velocity only for fastest $(q P 1)$ wave and the velocity change is largest (up to $8 \%$ ) for the slowest (qS2) wave. In the first column of the figure, the elevations and depressions of phasevelocity surfaces (from horizontal plane) measure the extent of velocity anisotropy in the medium. This anisotropy is quite significant but, nearly same for the three faster waves. However, velocity anisotropy is very small in case of the slowest wave. The anisotropic variations of the velocities are quite different for velocities obtained with different approaches. The only exception is the $q P 1$ wave where both the theories of Biot obtain nearly similar velocity variations. Attenuation is not there in this medium.

Medium of propagation, now, is anisotropic poroelastic solid saturated by a viscous fluid. The value of $\Delta_{o}=20$ is used to represent the lowfrequency regime of Biot's theory. From the plots of figure 1 , it is noted that the velocities obtained from Biot (1956) theory are quite different from the those obtained with Biot (1962a, b) theory. The reason being the differences in the inertial coupling in two theories. But, in case of dissipative medium, the viscous coupling dominates over the inertial coupling and, hence, the two theories of Biot obtain nearly the same velocities as well as attenuations for all the four waves. Hence, the variations of velocities and attenuations of the waves obtained from Biot (1956) theory are not plotted in the figures 2 and 3 . Figure 2 contains the variations of velocities of propagation $\left(V_{j}\right)$ with propagation direction, for each of the quasi-waves. The corresponding attenuations $\left(100 / Q_{j}\right)$ for the waves are plotted in figure 3 .

Comparison of figures 1 and 2 indicates that the presence of viscosity in the saturating fluid decreases the velocities of quasi-waves. The effect of the presence of viscosity is observed most on $q S 2$-wave, which is now propagating with only $30 \%$ of its velocity in figure 1 . The difference in the velocities of quasi-waves from Biot's theories and homogenisation is within $1 \%$ except the qP1wave. The velocity of this wave obtained from Biot's theory is up to $5 \%$ lesser than that obtained through homogenisation. The velocity anisotropies observed for three faster waves in figure 2 are of 
similar extent as in figure 1. In case of the slowest $(q S 2)$ wave, the velocity anisotropy is much larger in the dissipative medium.

The plots in the columns of figure 3 present the attenuations of quasi-waves due to the introduction of viscosity in the pore-fluid. The plots in the second column show that Biot's theory calculates up to $10 \%$ less attenuation for $q P 1$-waves. The gain in attenuation (up to $6 \%$ ) of $q P 2$-wave from Biot's theory is at the cost of $q S 1$-wave. Both the theories calculate larger attenuations for $q S 2$-waves. However, the difference in these attenuations is less than $1 \%$.

\section{Conclusions}

The mathematical expressions and relations derived in the text are general in nature whereas the numerical results are obtained for a particular model. So, a conclusion from the numerical part may not be significant quantitatively but the discussion of numerical results/plots reflects the qualitative aspects of different theories used. Also, the calculation of numerical results does certify the use of analytical expressions for the simulation of real data. Following are the few conclusions drawn from the study.

- The two theories of Biot and homogenisation theory for wave propagation in poroelastic solids are represented by the modified Christoffel equations and a relation among the displacement of two constituents of the porous aggregate.

- The three theories are represented by different and theoretically incompatible derivations. The differences may appear in the velocities, attenuations and polarizations of the quasi-waves propagating in the medium.

- Biot (1962a, b) theory, an advanced version of Biot (1956) theory, affects the propagation characteristics through the anisotropic inertial coupling between solid and fluid motions. The results are numerically different velocities, from the two theories of Biot, for propagation in nondissipative porous solid. However, in dissipative porous solid, due to the dominance of viscous coupling over inertial coupling, the effect of inertial coupling does not matter much. Hence, both the theories of Biot obtain almost same values for velocities as well as attenuations of all the four quasi-waves.

- The numerical results from the chosen numerical model suggest that in the absence of dissipation the homogenisation approach is providing almost the same output as that from Biot (1956) theory.

- The results from the numerical example may be used to conclude that the differences in velocities from these theories are not very serious and hence, can be ignored for practical purposes. The difference in attenuations is a bit significant.

- The anisotropic variations of velocity and attenuation are found to be depending upon the approach used. The differences in the numerical results from different theoretical approaches can be used to decide the application superiority of one approach over the other.

- The presence of viscosity in the interstitial fluid may slow down the wave propagation, in general. The major effect of viscosity on velocity is borne by the slowest $(q S 2)$ wave.

This study considers the three-dimensional wave propagation in a general anisotropic poroelastic medium. This is a mathematical study with little or no changes in a general physical model of a wave propagation problem. The three different descriptions are used to derive the mathematical models for wave propagation in the poroelastic medium. The purpose may be to analyze the role of description chosen on the propagation characteristics, quantitatively. Such an analysis may provide some guidelines for researchers in the field to prefer one description to the other. For example, the little differences among the numerical results from these descriptions may imply that a description with a comparatively simple mathematical model may be preferred over others. In other words, the use of the analytically simple homogenisation approach may not lead to much loss in comparison with Biot's theories for the wave propagation in saturated anisotropic porous solids. The study may be extended further to discuss the propagation of inhomogeneous waves in (visco) elastic porous solids. The mathematical models derived may become a part of the non-destructive testing/evaluation studies to understand the mechanical behaviour of composite materials (Braga 1990; Buden and Datta 1990; Wu and Wu 2000). The researchers in this field may find some interest in this study. The analytic equations derived in the work may be of some use for exploration or prospecting seismologists.

\section{References}

Auriault J L 1980 Dynamic behaviour of a porous medium saturated by a Newtonian fluid; Int. J. Engng. Sci. 18 $775-785$.

Auriault J L 1991 Dynamic behaviour of porous materials; In: Transport processes in porous media (eds) Bear J and Corapcioglu M Y (Kluwer) pp. 471-519.

Auriault J, Borne L and Chambon R 1985 Dynamics of porous saturated media: checking of generalized law of Darcy; J. Acoust. Soc. Am. 77 1641-1650.

Berryman J G 1980 Confirmation of Biot's theory; Appl. Phys. Lett. 37 382-384. 
Berryman J G and Wang H F 2001 Dispersion in poroelastic systems; Phys. Rev. E 6410.1103.

Biot M A 1956 The theory of propagation of elastic waves in a fluid-saturated porous solid, I. Low-frequency range, II. Higher frequency range; J. Acoust. Soc. Am. 28 168-191.

Biot M A 1962a Mechanics of deformation and acoustic propagation in porous media; J. Appl. Phys. 33 $1482-1498$.

Biot M A 1962b Generalized theory of acoustic propagation in porous dissipative media; J. Acoust. Soc. Am. 34 $1254-1264$.

Boutin C, Bonnet G and Bard P Y 1987 Green functions and associated sources in infinite and stratified media; Geophys. J. R. Astr. Soc. 90 521-550.

Boyle F A and Chotiros H P 1992 Experimental detection of a slow elastic wave in sediment at shallow grazing angles; J. Acoust. Soc. Am. 91 2615-2619.

Braga A M B 1990 Wave Propagation in Anisotropic Layered Composites; Ph.D. dissertation (Stanford University, Stanford, CA).

Buden M and Datta S K 1990 Rayleigh and love waves in cladded anisotropic media; ASME, J. Appl. Mech. $\mathbf{5 7}$ 398-403.

Burridge R and Keller J B 1981 Poroelasticity equations derived from microstructure; J. Acoust. Soc. Am. $\mathbf{7 0}$ $1140-1146$.

Carcione J M and Quiroga-Goode G 1996 Full frequencyrange transient solution for compressional waves in a fluid-saturated viscoacoustic porous medium; Geophys. Prosp. 44 99-129.

Chen J 1994 Time domain fundamental solution to Biot's complete equations of dynamic poroelasticity, part I: twodimensional solution; Int. J. Solids Struct. 31 1447-1490.

Crampin S 1989 Suggestions for a consistent terminology for seismic anisotropy; Geophys. Prospect. 37 753-770.

Gurevich B, Kelder O and Smeulders D M J 1999 Numerical simulation of ultrasonic experiments on poroelastic samples; Transport in Porous Media 36 149-160.

Hudson J A 1980 Overall properties of a cracked solid; Math. Proc. Camb. Phil. Soc. 88 371-384.

Hudson J A 1981 Wave speeds and attenuation of elastic waves in materials containing cracks; Geophys. J. R. Astr. Soc. 64 133-150.

Kaynia A M and Banerjee P K 1993 Fundamental solutions of Biot's equations of dynamic poroelasticity; Int. J. Engng. Sci. 31 817-830.
Kelder O and Smeulders D M J 1997 Observations of the Biot slow wave in water saturated Nivelsteiner sandstone; Geophysics 62 1794-1796.

King M S and Marsden J R 2002 Velocity dispersion between ultrasonic and seismic frequencies in brine-saturated reservoir sandstones; Geophysics 67 254-258.

King M S, Marsden J R and Dennis J W 2000 Biot dispersion for $P$ - and $S$-wave velocities in partially and fullysaturated sandstones; Geophys. Prosp. 48 1075-1089.

Klimentos T and McCann C 1988 Why is the Biot slow compressional wave not observed in real rocks?; Geophysics 53 1605-1609.

Lakes R, Yoon H S and Katz J L 1983 Slow compressional wave propagation in wet human and bovine cortical bone; Science 220 513-515.

Nagy P B, Alder L and Bonner B P 1990 Slow wave propagation in air-filled porous materials and natural rocks; Appl. Phys. Lett. 56 2504-2506.

Plona T J 1980 Observation of a second bulk compressional wave in a porous medium at ultrasonic frequencies; $A p p l$. Phys. Lett. 36 259-261.

Plona T J and Johnson D L 1984 Acoustic properties of porous systems: I. Phenomenological description; In: Physics and Chemistry of Porous Media (eds) Johnson D L and Sen P N (Am. Inst. of Physics, NY) pp. 89-104.

Pride S R, Berryman J G and Harris J M 2004 Seismic attenuation due to wave induced flow; J. Geophys. Res. 109 B01201.

Rasolofosaon P N J and Zinszner B E 2002 Comparison between permeability anisotropy and elasticity anisotropy of reservoir rocks; Geophysics 67 230-240.

Sharma M D 2004 Wave propagation in a general anisotropic poroelastic medium with anisotropic permeability: phase velocity and attenuation; Int. J. Solids \& Struct. 41 $4587-4597$.

Sharma M D 2005 Propagation of inhomogeneous plane waves in dissipative anisotropic porouelastic solids; $\mathrm{Geo}$ phys. J. Int. 163 981-990.

Sharma M D 2006 Wave propagation in anisotropic generalized thermoelastic media; J. Therm. Stresses 29 629-641.

Winkler K W 1985 Dispersion analysis of velocity attenuation in Berea sandstone; J. Geophys. Res. $906793-6800$.

$\mathrm{Wu} \mathrm{T}-\mathrm{T}$ and $\mathrm{Wu} \mathrm{T}-\mathrm{Y} 2000$ Surface waves in coated anisotropic medium loaded with viscous fluid; $A S M E$, J. Appl. Mech. 67 262-266. 